\begin{tabular}{|c|c|}
\hline Title & Electrical conductivity of carbon-nanotube/cellulose composite paper \\
\hline Author(s) & Tanaka, Tomo; Sano, Eiichi; Imai, Masanori; A kiyama, Kousuke \\
\hline Citation & $\begin{array}{l}\text { Journal of A pplied Physics, 107(5), } 054307 \\
\text { https://doi.org/10.1063/1.3319675 }\end{array}$ \\
\hline Issue Date & 2010-03-01 \\
\hline Doc URL & http:/hdl.handle.net/2115/42894 \\
\hline Rights & $\begin{array}{l}\text { Copyright } 2010 \text { A merican Institute of Phy sics. This article may be downloaded for personal use only. Any other use } \\
\text { requires prior permission of the author and the A merican Institute of Physics. The following article appeared in J. A ppl. } \\
\text { Phys. } 107,054307 \text { (2010) and may be found at https://dx.doi.org/10.1063/1.3319675 }\end{array}$ \\
\hline Type & article \\
\hline File Information & JAP107-5_054307.pdf \\
\hline
\end{tabular}

Instructions for use 


\title{
Electrical conductivity of carbon-nanotube/cellulose composite paper
}

\author{
Tomo Tanaka, ${ }^{1, a)}$ Eiichi Sano, ${ }^{1}$ Masanori Imai, ${ }^{2}$ and Kousuke Akiyama ${ }^{2}$ \\ ${ }^{1}$ Research Center for Integrated Quantum Electronics, Hokkaido University, Sapporo, Hokkaido 060-8628, \\ Japan \\ ${ }^{2}$ Tokushu Paper Co. Ltd., Nagaizumi, Shizuoka 411-8750, Japan
}

(Received 18 December 2009; accepted 18 January 2010; published online 4 March 2010)

We fabricated multiwalled carbon-nanotube/cellulose composite papers and measured their temperature dependences of electrical conductivity. The dependences were described with the Sheng's fluctuation-induced tunneling (FIT) model. A possible mechanism of the electrical conduction in the composite paper was discussed in the context of the FIT model.

(C) 2010 American Institute of Physics. [doi:10.1063/1.3319675]

\section{INTRODUCTION}

Carbon-nanotubes (CNTs) are considered to be a new class of material with reduced dimensionality. They have unique properties such as high mechanical strength, as well as high thermal and electrical conductivity. Much work has been done on CNT composites as well as on CNT sheets to fully utilize these properties for many kinds of applications. $^{1-3}$ To control their electrical conductance, the electrical conduction mechanism of CNT composites needs to be elucidated, and, to do this, the temperature dependences of electrical conductivities for CNT composites are very useful. ${ }^{4,5}$

There are two major mechanisms of electrical conduction for composite materials: variable range hopping (VRH) (Ref. 6) and fluctuation-induced tunneling (FIT). ${ }^{7}$ The VRH model matches the electrical conductivities of single-walled (SW) CNT mats ${ }^{8}$ and networks, ${ }^{9,10}$ while the FIT model matches those of SWCNT mats, ${ }^{11,12}$ SWCNT bundles, ${ }^{13}$ and SWCNT/polymer composites. ${ }^{8}$ On the other hand, the FIT model matches the electrical conductivities of multiwalled (MW) CNT composites. ${ }^{14-20}$ The electrical conduction through the CNT-CNT junctions dominates the conductivity of CNT network in the FIT model. The tunnel width $w$ and barrier height $V_{0}$ can be estimated from two specific temperatures $T_{0}$ and $T_{1}$ obtained by fitting the FIT law to the measured temperature dependence of the conductivity. However, only a few papers reported $w$ and $V_{0}$. Kim et al. ${ }^{11}$ estimated a $w$ of $3.5 \mathrm{~nm}$ and a $V_{0}$ of $0.1 \mathrm{eV}$ for a SWCNT network, while Mdarhri et al. ${ }^{19}$ estimated a $w$ of $5 \mathrm{~nm}$ and a $V_{0}$ of $15 \mathrm{meV}$ for MWCNT composites. These heights and widths seem to be too low and wide to be clearly explained. Salvato et al. $^{13}$ reported the barrier height dependence on the current and temperature in SWCNT bundles. The physical reason of this phenomenon is still unclear.

We report the temperature dependence of electrical conductivities for recently fabricated MWCNT/cellulose composite paper, a new kind of CNT composite, ${ }^{21}$ and discuss the possible conduction mechanism obtained by using rigorous calculations of $T_{0}$ and $T_{1}$ in the FIT model.

\section{FABRICATION AND MEASUREMENT METHODS}

The CNTs used in this work were MWCNTs, the Nanocyl 7000, fabricated by Nanocyl SA. The average diameter of the CNTs was $10 \mathrm{~nm}$, and the average length was $1.5 \mu \mathrm{m}$. A $1 \%$-CNT water dispersion was provided by Daido Corporation. Anionic surfactant was used to disperse CNTs. ${ }^{22}$ Pulp fibers have negative charges because they generate carboxyl groups during the cooking and bleaching involved in making paper making. ${ }^{23}$ The Coulomb repulsion prevents CNTs from fixing on pulp fibers. Therefore, a cationic fixer should be applied to fix the CNTs to the pulp.

Bleached hardwood Kraft pulp (50 wt \%) and bleached softwood Kraft pulp (50 wt \%) were dispersed in water and beat was obtained. A fixer of $2 \%$-cationized starch water solution was then added to the pulp and mixed with the $1 \%$ CNT dispersion. Hand-made CNT/cellulose composite paper was prepared using a $25 \times 25 \mathrm{~cm}^{2}$ wire cloth. A scanning electron microscope (SEM) image is shown in Fig. 1. A CNT network was formed on the surface of cellulose fiber.

We prepared three samples of paper with different CNT contents of 1,4 , and 5 wt $\%$. The CNT content was calculated by dividing the weight of CNTs by the total weight of CNTs and cellulose because it could not be determined by thermogravimetric analysis with sufficient accuracy. The thickness of the paper was $0.15 \mathrm{~mm}$.

The electric conductivity was measured by a four-probe method with a source meter (Keithley 2636A). Small pieces of indium were bonded to the samples to ensure that there

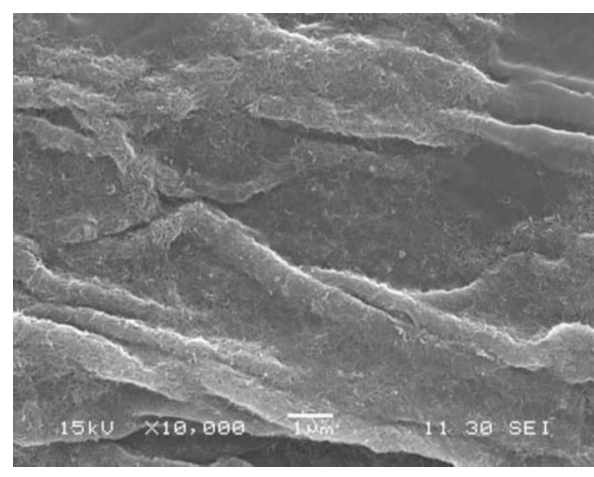

FIG. 1. SEM image of MWCNT/cellulose composite paper.

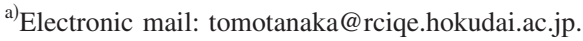




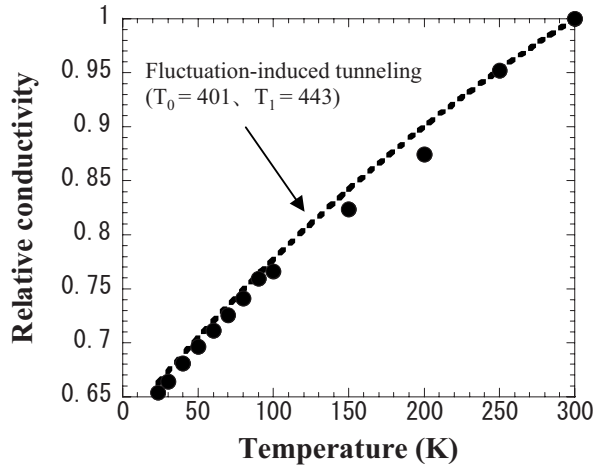

FIG. 2. Measured temperature dependence of electrical conductivity normalized with conductivity at $300 \mathrm{~K}$.

was proper contact with the probe. The current was increased from 0 to $0.1 \mathrm{~mA}$ to check the linearity of current-voltage characteristics. The temperature was increased from 10 to $300 \mathrm{~K}$.

\section{RESULTS AND DISCUSSION}

The temperature dependences of electrical conductivity normalized with the conductivity at $300 \mathrm{~K}$ for the composite paper with a CNT content of 1 wt $\%$ are shown in Fig. 2. According to the Sheng's FIT model, ${ }^{7}$ the conductivity is expressed as

$$
\sigma=\sigma_{0} \exp \left(-\frac{T_{1}}{T+T_{0}}\right)
$$

where $T_{0}$ and $T_{1}$ are the specific temperatures. The measured temperature dependence of electrical conductivity can be fitted to the FIT model with $T_{0}=401 \mathrm{~K}$ and $T_{1}=443 \mathrm{~K}$ as shown by the dotted line in Fig. 2. The same procedure revealed that $T_{0}=602 \mathrm{~K}$ and $T_{1}=543 \mathrm{~K}$ for $4 \mathrm{wt} \%$ and $T_{0}$ $=105 \mathrm{~K}$ and $T_{1}=192 \mathrm{~K}$ for $5 \mathrm{wt} \%$ composite papers.

The $T_{0}$ and $T_{1}$ obtained from our measurements with those reported in Refs. 8, 11, 13-17, and 20 are shown in Fig. 3. The probability for tunneling at the Fermi energy through a junction between two CNTs is proportional to $\exp \left(-T_{1} / T_{0}\right)$. The dotted lines in Fig. 3 correspond to constant tunneling probabilities. Our $T_{0}$ and $T_{1}$ were close to those for SWCNT composites ${ }^{8}$ and MWCNT composites. ${ }^{14,16,20}$ As mentioned before, the tunnel width $w$

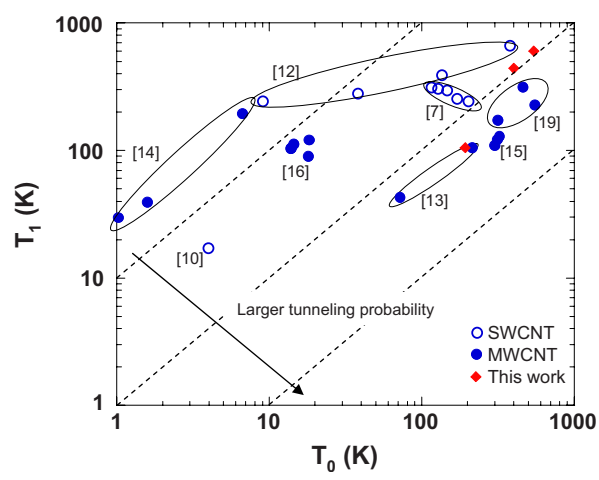

FIG. 3. (Color online) Comparison of our $T_{0}$ and $T_{1}$ with those reported in literature (Refs. 8, 11, 13-17, and 20) Dotted lines correspond to constant tunneling probabilities.

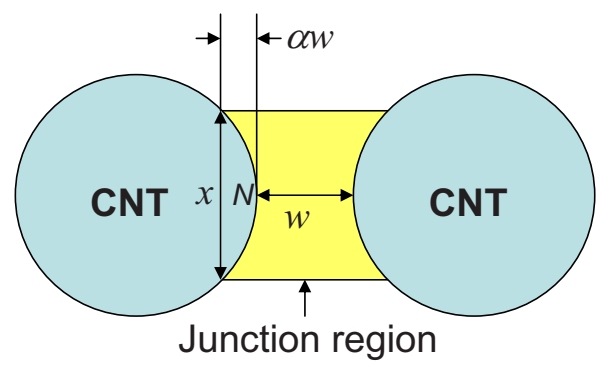

FIG. 4. (Color online) Cross section of junction model used for calculations.

and barrier height $V_{0}$ can be estimated from $T_{0}$ and $T_{1}$. Kim et al. ${ }^{11}$ and Mdarhri et al. ${ }^{19}$ used approximate expressions for $T_{0}$ and $T_{1}$. Since there are three unknown parameters $\left(w, V_{0}\right.$, and the junction area $A$ ) in the two equations, we cannot determine these values uniquely. Applying the equations to our values of $T_{0}$ and $T_{1}$ leads to $w V_{0}^{1 / 2}=1.37 \times 10^{-10}$. When we assume $V_{0}$ to be $4.4 \mathrm{eV}$ [the work function of MWCNT (Ref. 24)], $w$ becomes $0.065 \mathrm{~nm}$. This is an unrealistic value because it is smaller than the distance between two carbon atoms in the hexagonal cell.

We then estimate $w, V_{0}$, and $A$ on the basis of a junction model and rigorous calculations of Sheng's model. According to the FIT model, ${ }^{7}$ the specific temperatures are

$$
\begin{aligned}
& T_{1}=a \varepsilon_{0}^{2} / k, \\
& T_{0}=T_{1} / 2 x w \xi(0),
\end{aligned}
$$

where $a=w A / 8 \pi$ is the junction volume, $w$ is the tunnel width, $A$ is the junction area, $k$ is the Boltzmann constant, and $x=\left(2 m V_{0} / \hbar^{2}\right)^{1 / 2}$ is the tunneling constant. Here, $\xi(0)$ is defined as follows:

$$
\xi(0)=\int_{u_{3}}^{u_{4}}\left[1-\frac{\lambda}{u(1-u)}\right]^{1 / 2} d u,
$$

where $u_{3}, u_{4}\left(>u_{3}\right)$ denotes the two zeros of potential $V(u, \varepsilon)$, and $\lambda=0.795 e^{2} / 4 w K V_{0}$ with $K$ being the dielectric constant of the barrier region. In (2), $\varepsilon_{0}$ is the electric field at which the potential maximum equals to 0 . Refer to Sheng's paper ${ }^{7}$ for more detail.

A crosssection of the junction region we used as a model is shown in Fig. 4. The CNT surface belonging to the junction region is defined as an arc whose ends are laterally apart from the point $N$ by $\alpha w$. The junction area $A$ is defined as $x^{2}$. The diameter of CNT was assumed to be $10 \mu \mathrm{m}$. The dielectric constant of the barrier region $K$ was assumed to be 1 .
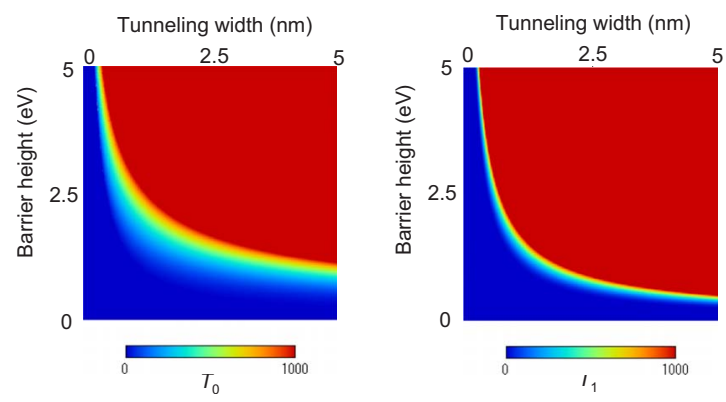

FIG. 5. (Color online) Example of calculated $T_{0}$ and $T_{1}$ in $w-V_{0}$ plane. 


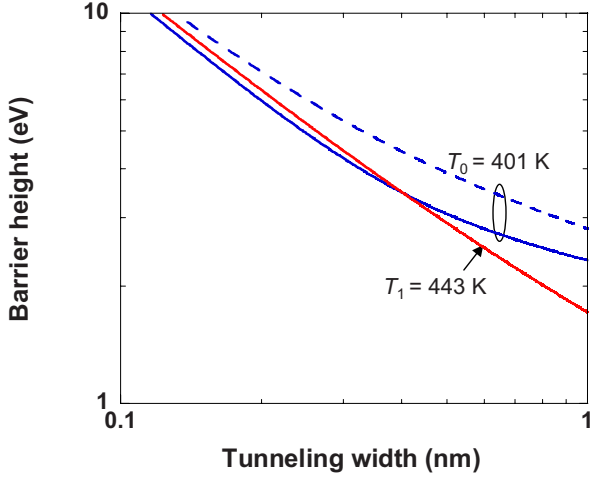

FIG. 6. (Color online) Magnified view of Fig. 5.

An example of calculated $T_{0}$ and $T_{1}$ in the $w-V_{0}$ plane Fig. 5 . A magnified view of Fig. 5 is shown in Fig. 6. The intersection of the two contours for $T_{0}$ and $T_{1}$ gives a set of solution for $w$ and $V_{0}$. The dotted line for $T_{0}$ shown in Fig. 6 was calculated by assuming that $\xi(0)=1$. The contour for $T_{0}$ does not cross the contour for $T_{1}$ in the region shown in Fig. 6 . This means that an exact numerical calculation is needed to obtain a set of solution for $w$ and $V_{0}$.

Estimated $w$ and $V_{0}$ are summarized in Fig. 7, where the symbols denote $w$ and $V_{0}$ calculated with $T_{0}$ and $T_{1}$ described above and the regions enclosed by the dotted line using $a=0.01$ correspond to $w$ and $V_{0}$ that match the measured conductivity within $1 \%$ standard-deviation uncertainty. No distinct dependence of $w$ and $V_{0}$ on the CNT fraction was observed. The $w$ and $V_{0}$ were close to the interlayer distance of graphite $(0.34 \mathrm{~nm})$ and the work function of MWCNT (4.4 $\mathrm{eV})$. The uncertainty ranges for $w$ and $V_{0}$ are very narrow. Since almost all the surfactant had been removed in the paper making process and the sizes of cellulose fibers were much larger than the estimated $w$, two CNTs are thought to form a junction with no inclusion. The electrical conduction described in our composite paper was dominated by such CNT-CNT junctions. Although the junction model is simple, this is a reasonable consequence. Fuhrer et $\mathrm{al}^{25}$ used the first principles method to calculate the tunneling probability of CNT-CNT junction with a distance of $0.34 \mathrm{~nm}$ to be 2 $\times 10^{-4}$. The narrow region between CNTs acts as a potential

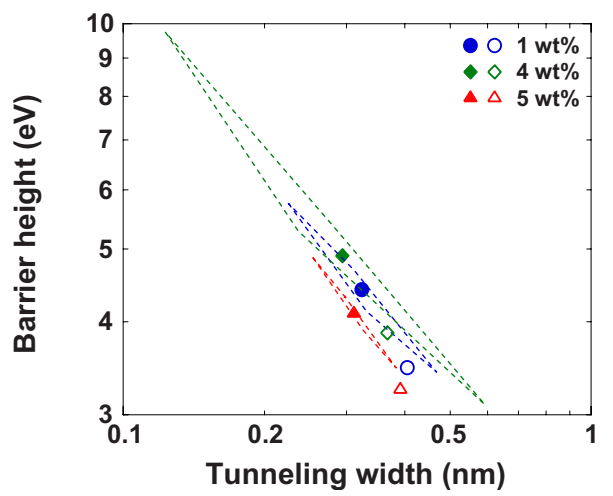

FIG. 7. (Color online) Estimated $w$ and $V_{0}$. Solid and open symbols are calculated with $\alpha=0.01$ and 0.02 , respectively, from $T_{0}$ and $T_{1}$ that optimally fit measured data to FIT model. Regions enclosed by dotted line for $\alpha=0.01$ correspond to $w$ and $V_{0}$ that reproduce measured temperature dependence of the conductivity within $1 \%$ standard deviation.

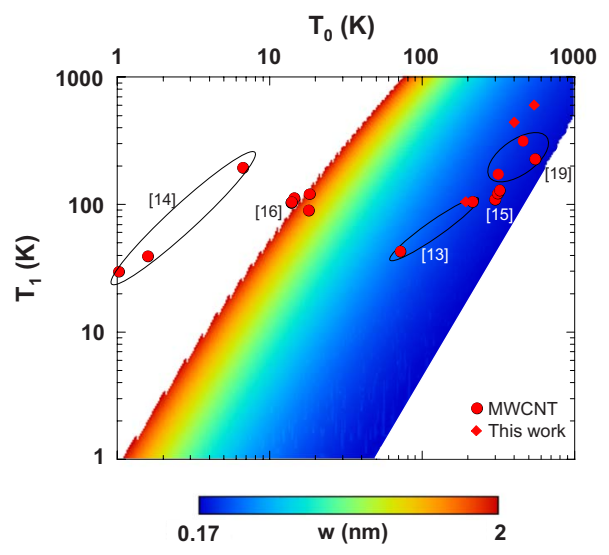

FIG. 8. (Color online) Map of tunneling width $w$ in $T_{0}-T_{1}$ plane.

barrier. It is quite natural to think that $V_{0}$ equals the work function of MWCNT. The calculation with $\alpha=0.01$ suggests that $w$ was around $0.3 \mathrm{~nm}$ smaller than the interlayer distance of graphite. Crossed MWCNTs might be deformed at the junction as calculated by the first principles method. ${ }^{25,26}$

A map of the tunneling width $w$ in the $T_{0}-T_{1}$ plane is shown in Fig. 8. Here, the barrier height $V_{0}$ was decreased from 25 to $5 \mathrm{eV}$. $\alpha=0.01$ and $K=1$. The $T_{0}$ and $T_{1}$ reported in the literature ${ }^{14-17,20}$ also are plotted in Fig. 8. It is noted that the exact area of the junction should be calculated with the first principles method and the accuracy of the map should be improved in future work. However, the present map of the tunneling width $w$ may be useful for qualitatively elucidating the properties of CNT networks.

\section{CONCLUSION}

In summary, we fabricated MWCNT/cellulose composite papers and measured their temperature dependences of electrical conductivity. The dependences were described with the Sheng's FIT model. The tunneling widths and barrier heights were estimated from the specific temperatures $T_{0}$ and $T_{1}$ deduced from rigorous calculations with a simple junction model. The estimated widths and barrier heights were close to the interlayer distance of graphite and the work function of MWCNT. This suggested that the electrical conduction in MWCNT/cellulose composite paper was dominated by the FIT between the CNT-CNT junctions and there was no inclusion.

${ }^{1}$ M. Zhang, S. Fang, A. A. Zakhidov, S. B. Lee, A. E. Aliev, C. D. Williams, K. R. Atkinson, and R. H. Baughman, Science 309, 1215 (2005).

${ }^{2}$ G. Gruner, J. Mater Chem. 16, 3533 (2006).

${ }^{3}$ Q. Cao and J. A. Rogers, Adv. Mater. 21, 29 (2009).

${ }^{4}$ A. B. Kaiser, Adv. Mater. 13, 927 (2001)

${ }^{5}$ A. N. Aleshin, Adv. Mater. 18, 17 (2006).

${ }^{6}$ N. F. Mott and G. A. Davis, Electronic Properties in Noncrystalline Materials, 2nd ed. (Clarendon, Oxford, 1979).

${ }^{7}$ P. Sheng, Phys. Rev. B 21, 2180 (1980).

${ }^{8}$ E. Kymakis and G. A. J. Amaratunga, J. Appl. Phys. 99, 084302 (2006). ${ }^{9}$ V. Skákalová, A. B. Kaiser, Y.-S. Woo, and S. Roth, Phys. Rev. B 74, 085403 (2006).

${ }^{10}$ M. Jaiswal, W. Wang, K. A. Shiral Fernando, Y.-P. Sun, and R. Menon, J. Phys.: Condens. Matter 19, 446006 (2007).

${ }^{11}$ G. T. Kim, S. H. Jhang, J. G. Park, Y. W. Park, and S. Roth, Synth. Met. 117, 123 (2001).

${ }^{12}$ M. Shiraishi and M. Ata, Synth. Met. 128, 235 (2002). 
${ }^{13}$ M. Salvato, M. Cirillo, M. Lucci, S. Orlanducci, I. Ottaviani, M. L. Terranova, and F. Toschi, Phys. Rev. Lett. 101, 246804 (2008).

${ }^{14}$ H. M. Kim, K. Kim, C. Y. Lee, J. Joo, S. J. Cho, H. S. Yoon, D. A. Pejakovic, J. W. Yoo, and A. J. Epstein, Appl. Phys. Lett. 84, 589 (2004). ${ }^{15}$ H. M. Kim, M.-S. Choi, J. Joo, S. J. Cho, and H. S. Yoon, Phys. Rev. B 74 054202 (2006).

${ }^{16}$ S.-L. Shi and J. Liang, J. Appl. Phys. 101, 023708 (2007).

${ }^{17}$ R. Zhang, M. Baxendale, and T. Peijs, Phys. Rev. B 76, 195433 (2007).

${ }^{18}$ Y. Simsek, L. Ozyuzer, A. T. Seyhan, M. Tanoglu, and K. Schulte, J. Mater. Sci. 42, 9689 (2007).

${ }^{19}$ A. Mdarhri, F. Carmona, C. Brosseau, and P. Delhaes, J. Appl. Phys. 103, 054303 (2008).
${ }^{20}$ K. Ahmad and W. Pan, Compos. Sci. Technol. 69, 1016 (2009).

${ }^{21}$ M. Imai, K. Akiyama, T. Tanaka, and E. Sano (unpublished).

${ }^{22}$ c8Y. Morimoto and N. Kubosaki, Japan Patent Kokai 2007-157440 (2007).

${ }^{23}$ M. Kato, A. Isogai, and F. Onabe, J. Wood Sci. 44, 361 (1998).

${ }^{24}$ H. Ago, T. Kugler, F. Cacialli, W. R. Salaneck, M. S. P. Shaffer, A. H. Windle, and R. H. Friend, J. Phys. Chem. B 99, 103 (1999).

${ }^{25}$ M. S. Fuhrer, J. Nygard, L. Shih, M. Forero, Y.-G. Yoon, M. S. C. Mazzoni, H. J. Choi, J. Ihm, S. G. Louie, A. Zettl, and P. L. McEuen, Science 288, 494 (2000).

${ }^{26}$ T. Hertel, R. E. Walkup, and P. Avouris, Phys. Rev. B 58, 13870 (1998). 